\title{
Eight versus 28-point lung ultrasonography in moderate acute heart failure: a prospective comparative study
}

\author{
Antonio Leidi ${ }^{1}$ (1) $\cdot$ Guillaume Soret $^{1} \cdot$ Tamara Mann $^{1} \cdot$ Flora Koegler $^{1} \cdot$ Matteo Coen $^{1,2} \cdot$ Alexandre Leszek $^{1}$. \\ Laetitia Dubouchet ${ }^{1}$ - Alexandre Guillermin ${ }^{1} \cdot$ Myriam Kaddour $^{1}$. Frédéric Rouyer ${ }^{3}$. Christophe Combescure ${ }^{4}$. \\ Sebastian Carballo ${ }^{1}$. Jean-Luc Reny ${ }^{1}\left[\right.$. $\cdot$ Christophe Marti $^{1}$. Jérôme Stirnemann ${ }^{1}$. Olivier Grosgurin ${ }^{1,3}$
}

Received: 13 October 2021 / Accepted: 1 February 2022 / Published online: 18 February 2022

(c) The Author(s) 2022

\begin{abstract}
Lung ultrasonography (LUS) is an accurate method of estimating lung congestion but there is ongoing debate on the optimal number of scanning points. The aim of the present study was to compare the reproducibility (i.e. interobserver agreement) and the feasibility (i.e. time consumption) of the two most practiced protocols in patients hospitalized for acute heart failure (AHF). This prospective trial compared 8- and 28-point LUS protocols. Both were performed by an expert-novice pair of sonographers at admission and after 4 to 6 days on patients admitted for AHF. A structured bio-clinical evaluation was simultaneously carried out by the treating physician. The primary outcome was expert-novice interobserver agreement estimated by kappa statistics. Secondary outcomes included time spent on image acquisition and interpretation. During the study period, 43 patients underwent a total of 319 LUS exams. Expert-novice interobserver agreement was moderate at admission and substantial at follow-up for 8-point protocol (weighted kappa of 0.54 and 0.62 , respectively) with no significant difference for 28-point protocol (weighted kappa of 0.51 and $0.41 ; P$ value for comparison 0.74 at admission and 0.13 at follow-up). The 8-point protocol required significantly less time for image acquisition at admission (mean time difference $-3.6 \mathrm{~min}$ for experts, $-5.1 \mathrm{~min}$ for novices) and interpretation $(-6.0 \mathrm{~min}$ for experts and $-6.3 \mathrm{~min}$ for novices; $P$ value $<0.001$ for all time comparisons). Similar differences were observed at follow-up. In conclusion, an 8-point LUS protocol was shown to be timesaving with similar reproducibility when compared with a 28-point protocol. It should be preferred for evaluating lung congestion in AHF inpatients.
\end{abstract}

Keywords Lung ultrasonography $\cdot$ Ultrasound $\cdot$ Heart failure $\cdot$ Congestion $\cdot$ Protocol $\cdot$ Eight

Jérôme Stirnemann and Olivier Grosgurin contributed equally to the study.

Antonio Leidi

Antonio.Leidi@hcuge.ch

1 General Internal Medicine, Department of Medicine, Geneva University Hospitals, Rue Gabrielle-Perret-Gentil 4, 1205 Geneva, Switzerland

2 Unit of Development and Research in Medical Education (UDREM), Faculty of Medicine, University of Geneva, Geneva, Switzerland

3 Emergency Medicine, Department of Acute Medicine, Geneva University Hospitals, Geneva, Switzerland

4 Clinical Research Center and Division of Clinical Epidemiology, Department of Health and Community Medicine, University of Geneva and Geneva University Hospitals, Geneva, Switzerland

\section{Introduction}

Despite therapeutic advances, acute heart failure (AHF) remains the leading cause of hospital admission and one of the most frequent reasons for readmission in northern countries [1-3]. As the main reason for AHF hospitalization is congestion-driven symptoms, the cornerstone of treatment is decongestive therapy [4]. In the absence of specific quantitative measures, however, residual congestion is noted at discharge in $10-15 \%$ of patients and is associated with an increased risk of readmission [5]. Lung ultrasonography (LUS) has been progressively incorporated into medical practice [6]. LUS has high level of accuracy for extravascular lung water detection (EVLW) and provides a semiquantification of congestion, even at subclinical stage [7, 8]. Decrease in B-lines, the sonographic hallmark of cardiogenic edema, correlates with clinical improvement and 


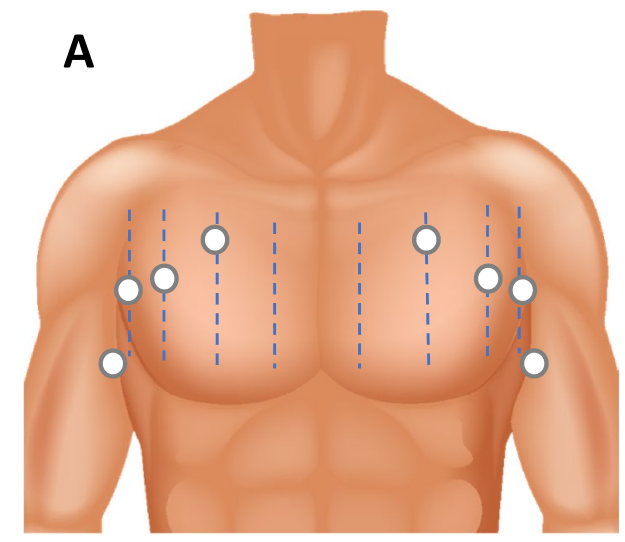

Fig. 1 Lung ultrasonography protocols. In eight-point protocol (Panel A) thorax is explored bilaterally in second intercostal space (ics) on mid-clavicular line (mcl), in forth ics on anterior axillary line (aal), in fifth ics on mid-axillary line (mal) and in the seventh ics beyond the

can be used to guide decongestion [9-12], whereas their persistence after treatment is associated with an increased risk of hospital admission [13-15].

The several existing protocols differ in exhaustiveness (i.e. number and localization of scanning points) and rating methodology [16]. Eight- and 28-point protocols are generally preferred when following patients suffering from heart failure [17]. Eight-point protocols seem to have similar diagnostic value but less time when performed at admission in emergency departments (ED) or intensive care units (ICU) [18, 19]. No comparative data exist for less congested patients, such as AHF inpatients. A short training period is sufficient to recognize B-lines; indeed the learning curve is known to be sharper than for other US techniques [20,21]. Nevertheless, in most studies only experienced sonographers performed and interpreted LUS, raising the question of generalizability of results.

The aim of the present study was to compare 8- and 28-point LUS protocols in terms of reproducibility (expertnovice interobserver agreement), feasibility (time for images acquisition and interpretation), and performance (correlation with clinical features and biomarkers).

\section{Methods}

The present article was written in accordance with the ESC reporting checklist for lung ultrasound studies in heart failure cohorts [22], the STROBE Statement checklist and was registered in clinicaltrial.gov (NCT 04,174,794). The investigation conforms with the principles outlined in the Declaration of Helsinki and was approved by the local ethics committee (CCER 2019-01,596). Informed consent was obtained from all patients prior to inclusion.

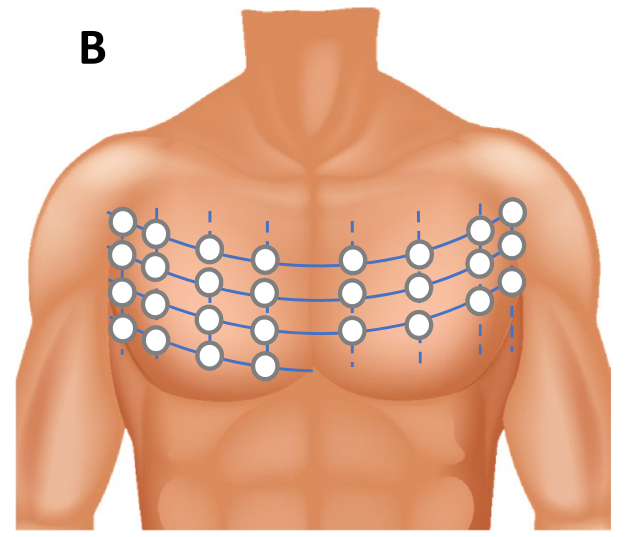

posterior axillary line. In twenty-eight-point protocol (Panel B) thorax is explored from the second to the fifth ics in right hemithorax and from the second to the fourth ics in left hemithorax, along four thoracic lines (parasternal line, mcl, aal, mal)

This single-center prospective observational study included adults hospitalized consecutively for AHF regardless of left ventricular ejection fraction. AHF was defined according to ESC criteria [4] (presence of $\geq 1$ sign or symptom and a value of $\mathrm{N}$-terminal-pro-B-type natriuretic peptide (NT-proBNP) of $\geq 300 \mathrm{ng} / \mathrm{l}$ ). Participants were included when both expert and novice sonographers were available. Patients admitted directly to ICU were excluded in addition to those with comorbidities known to produce B-line artefacts (i.e. interstitial lung diseases, ARDS, lung cancer or metastasis, lung contusion, previous lung surgery). Patients with oligo-anuric end stage kidney disease and unwillingness or inability to give consent were also excluded. To avoid unnecessary patient selection, a concomitant diagnosis of pneumonia was not considered an exclusion criterion, even if this condition can present with B-lines. Setting, recruitment and procedures are detailed in Appendix 1, 2.

\section{Lung ultrasonography}

All images were obtained with high-end devices; details on knobology and ultrasonography procedures are described in Appendix 3.

\section{Eight-point protocol}

This protocol was adapted from existing protocols [23, 24] and is represented in Fig. 1. The transducer was oriented in a sagittal plan to visualize one ICS and two ribs with their shadows. A 1 centimetre lateral translation of the probe in each direction was allowed to obtain a better acoustic window. Every point was coded $(p=1)$ in presence of $\geq 3$ B-lines simultaneously on a frozen image or in presence of pleural effusion. This was introduced as we considered 
pleural effusion as a marker of congestion. The total score ranged from 0 to 8 .

\section{Twenty-eight-point protocol}

In the 28-point protocol the thorax was scanned from the second to the fifth ICS in right hemithorax and from the second to the fourth ICS in left hemithorax, following four thoracic lines (Fig. 1). The sum of the maximum number of B-lines visualized on a frozen image for each scanning point yielded a score denoting the extent of the pulmonary congestion. According to the original description [25], the transducer was oriented in a transversal plan allowing a larger visualization of the pleural line. When visualization of B-lines was impeded by extra-pulmonary structures (e.g. heart) or pleural effusion, the affected point scored zero B-lines.

\section{Outcomes}

The primary outcome was interobserver agreement between expert and novice sonographers for both protocols. To compare protocols using different grading systems, results were rated with a common pre-specified 4-levels lung congestion scale (LCS). For the 28-point protocol, lung congestion was classified in accordance with the literature as severe $(>30)$, moderate (16-30), mild (6-15) or absent ( $\leq 5$ B-lines). The 8-point protocol was arbitrarily predefined as follows: severe (6-8), moderate (4-5), mild (2-3) and absent (0-1 positive points). To emphasize full decongestion, both LCS were additionally categorised in a dichotomous way (i.e. absence/ presence of congestion) using a cut-off of $\leq 5$ B-lines and $\leq 1$ positive points for the 28 -point and 8 -point protocols, respectively. As secondary outcomes, we measured the time spent for image acquisition and for interpretation. Additionally, change in B-lines after decongestive therapy was analyzed by computing admission-follow-up difference in LCS. It was subsequently correlated with the EVEREST score, body weight and NT-proBNP evolution. Finally, we explored the relationship between aLUS and length of stay as well as fLUS and short-term readmission and mortality.

\section{Potential sources of bias}

Sonographer competence, patient body mass index (BMI), time since diuretic administration, patient position, ultrasound device, knobology and image processing could potentially impact the B-lines count. To limit the influence of part of these variables, LUS scans were executed within a 60 min timespan by both expert and novice, the patient lying in a pre-determined position (see Appendix 3). In addition, standardized US device, probe and image processing were used. Moreover, the primary outcome was estimated posthoc in a sub-group of obese patients (BMI $\geq 30 \mathrm{~kg} / \mathrm{m}^{2}$ ).

\section{Statistical analysis}

In this exploratory study, a sample of 90 patients was initially planned to obtain a precision in estimate of kappa statistic around \pm 0.12 . However, due to recruitment suspension in March, 2020 in non-SARS-CoV-2 related studies due to cross-infection risks, 43 patients were in fact recruited. Characteristics of participants are presented with descriptive statistics with median and interquartile range for continuous variables and percentages for categorical variables. Expert-novice interobserver agreement was estimated by kappa statistics, with Cicchetti-Allison's weighting. Differences in agreement between 8- and 28-point protocols were assessed independently at admission and follow-up using a permutation test. For US image acquisition and interpretation time differences, outcome comparison was conducted by paired $t$ test. Length of stay and early readmission and mortality were compared with Wilcoxon rank test and Fisher's exact test, respectively. A 2 -sided $p$ value of $<0.05$ has been considered to infer statistical significance. Spearman correlation coefficient was used to assess correlation between evolution in LUS scores and bio-clinical variables between admission and follow-up; NT-proBNP delta was expressed in percentage. A post-hoc analysis was performed to assess correlation between LUS and bio-clinical congestion markers at admission and follow-up, separately. No replacement of missing data was planned.

\section{Results}

Between October 8th, 2019 and March 16th, 2020, 43 patients (mean age of 76 years, $26 \%$ of women, mean left ventricular ejection fraction of $43 \%$ ) underwent up to 8 LUS exams for a total of 319, 162 performed by three expert and 157 by ten novice sonographers. All subjects had at least one aLUS and four (9\%) had no fLUS due to unplanned early hospital discharge or the absence of B-lines on aLUS (Fig. 2). For approximately half of the patients this was their first hospitalization for heart failure and less than half of all patients had ejection fraction $<40 \%$ (Table 1 ). At inclusion, almost all patients (93\%) presented signs of peripheral congestion (i.e. lower limbs oedema or lung rales) on physical examination but $20 \%$ showed no signs of pulmonary congestion on auscultation (Table 2).

Imaging was 100\% feasible for the 8-point protocol. In contrast, when performing the 28-point protocol, examination was impeded in $18 \%$ of scanning points by extrapulmonary structures (e.g. abdominal organs, pleural effusion, pace-makers). Admission LUS were performed on 
Fig. 2 Study flow diagram

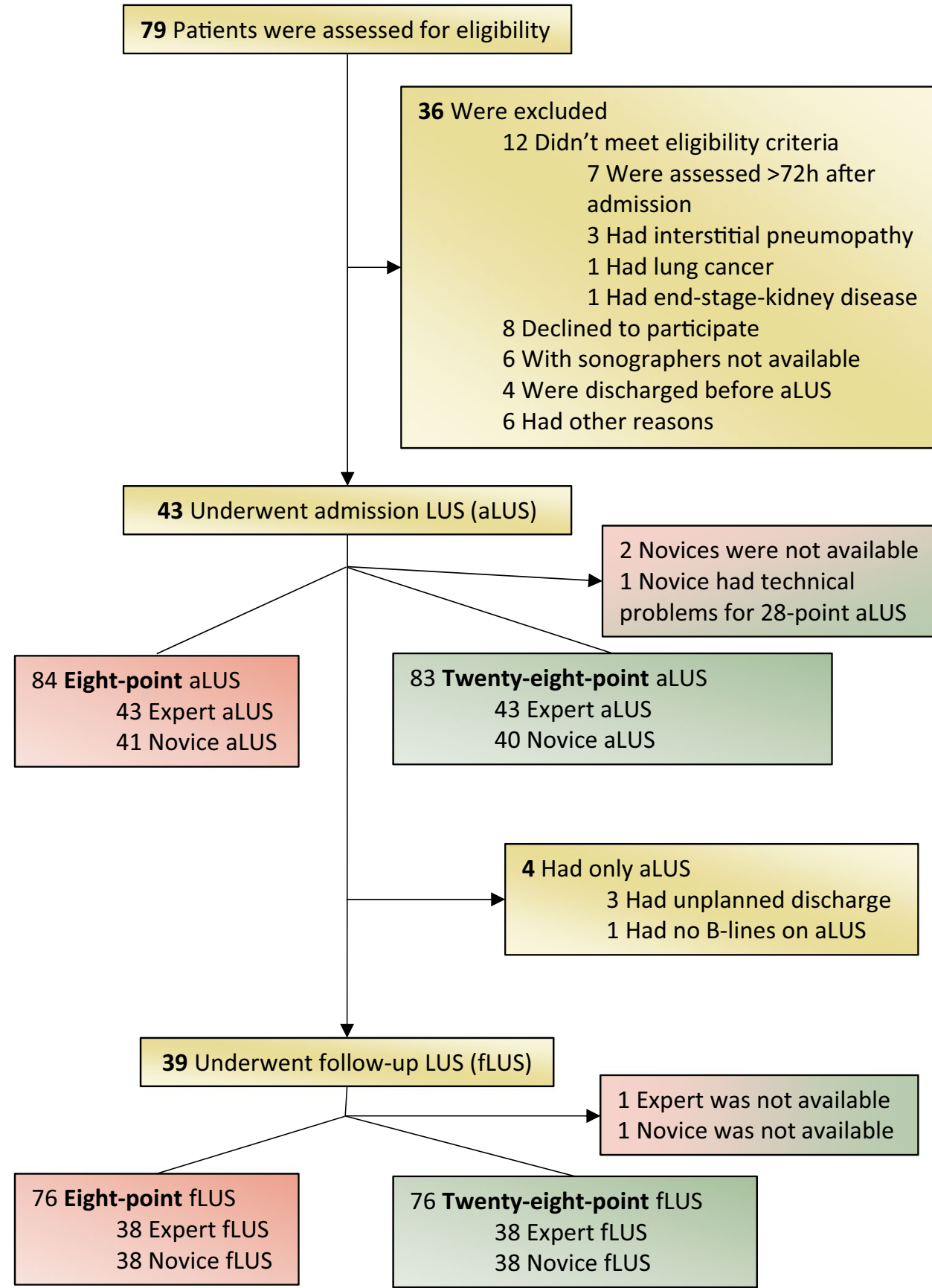

average 1 day (IQR 1 to 3 ) after admission to the ward. Significant pulmonary congestion was detected by experts at admission in 86 and $91 \%$ of subjects using the 8-point and 28-point protocols, respectively, whereas pleural effusion was present in $72 \%$ of subjects. Proportions were lower for novices $(67 \%, 91 \%, 50 \%$, respectively). Followup LUS was performed after a median period of 4.5 days (IQR 4 to 6). Only one patient had delayed fLUS (14 days) due to a rapid decline in clinical condition requiring ICU admission. For all protocols, scores decreased at fLUS:
20 and $28 \%$ relative decrease in LCS was observed using 8 -point protocol, 25 and $13 \%$ using 28-point protocol, for expert and novices, respectively (Appendix 4). Globally, congestion was more prevalent in lateral (particularly infero-lateral) than anterior zones (Appendix 5). For five patients a concomitant diagnosis of pneumonia was documented by treating physicians. Two patients needed unblinding and the communication of the expert aLUS results to the treating physician due to pre-specified potential life-threatening conditions as follows: absence of B-lines in a hypoxemic patient (potentially signalling 
Table 1 Baseline demographics and clinical characteristics of patients

\begin{tabular}{lc}
\hline & Total $(n=43)$ \\
\hline Age, median (IQR), years & $76(65-84)$ \\
Men, $n(\%)$ & $32(74)$ \\
BMI, median (IQR), kg/m ${ }^{2}$ & $27(24-32)$ \\
Medical history & \\
Prior heart failure, $n(\%)$ & $23(54)$ \\
Hypertension, $n(\%)$ & $31(72)$ \\
Diabetes mellitus, $n(\%)$ & $18(42)$ \\
Atrial fibrillation/flutter, $n(\%)$ & $20(47)$ \\
Valvulopathy, $n(\%)$ & $23(54)$ \\
CAD, $n$ (\%) & $16(37)$ \\
Chronic kidney disease, $n(\%)$ & $12(28)$ \\
Concomitant pneumonia, $n(\%)$ & $5(12)$ \\
Echocardiography & \\
LVEF, median (IQR), $\%$ & $43(27-60)$ \\
$\geq 50 \%, n(\%)$ & $19(44)$ \\
$40-49 \%, n(\%)$ & $6(14)$ \\
$<40 \%, n(\%)$ & $18(42)$ \\
Serum creatinine, median (IQR), $\mu$ mol/L & $109(87-142)$ \\
Serum hemoglobin, median (IQR), g/L & $131(104-145)$ \\
Serum albumin, median (IQR), g/L & $37(35-40)$ \\
Destination after discharge, $n(\%)$ & \\
Home & $33(77)$ \\
Rehabilitation & $8(19)$ \\
Other & $2(5)$ \\
\hline
\end{tabular}

$B M I$ means body mass index, $L V E F$ means left ventricular ejection fraction, $C A D$ means coronary artery disease

the presence of pulmonary embolism) and presence of asymmetric isolated lung consolidation (compatible with pneumonia). Discharge diagnoses were right heart failure and pneumonia, respectively.

Overall, the median length of stay was 13 days (IQR 5 to 17) with most patients being discharged home (77\%). Cumulative mortality and readmission rate at day 30 postdischarge was $16 \%$ ( 2 deaths and 5 readmissions). Proportions were higher at day 60 post-discharge (Total 23\%, mortality $5 \%$, readmissions $18 \%$ ).

\section{Primary outcome}

Expert-novice interobserver agreement was moderate at admission for both the 8-point (weighted kappa 0.54, 95\% CI 0.35 to 0.74$)$ and the 28 -point protocol $(0.51,95 \%$ CI 0.31 to 0.71 ). Substantial interobserver agreement was obtained for the 8-point protocol at follow-up $(0.62,95 \%$ CI 0.47 to 0.77 ), whereas it was moderate for the 28-point protocol $(0.41,95 \%$ CI 0.25 to 0.57$)$. However, the difference was not statistically significant $(P=0.74$ at admission and $P=0.13$ at follow-up). Results did not substantially differ in a subgroup of patients with BMI $\geq 30 \mathrm{~kg} / \mathrm{m}^{2}$ (Table 3) nor were they influenced by the increased experience of novice sonographers throughout the study (Appendix 6). Bland-Altman plots are available in Appendix 7.

\section{Secondary outcomes}

Image acquisition and interpretation time was significantly lower for the 8-point compared to 28-point protocol $(P<0.001$ for all comparisons). On average, the 8-point protocol required less than $3 \mathrm{~min}$ for experts (aLUS: $2.95 \mathrm{~min}$; fLUS: $2.8 \mathrm{~min}$ ) compared with more than the double that time for the 28-point (aLUS: $6.52 \mathrm{~min}$; fLUS: $6.23 \mathrm{~min}$ ); time difference $-3.6 \min (95 \% \mathrm{CI}-4.2$ to -3.0$)$ and -3.4 (95\% CI -4.1 to -2.8$)$ at admission and followup, respectively. Novices spent more than 4 min performing the 8-point (aLUS: $4.12 \mathrm{~min}$; fLUS: $4.7 \mathrm{~min}$ ), and at least $9 \mathrm{~min}$ for the 28-point protocol (aLUS: $9.32 \mathrm{~min}$; fLUS: $9.02 \mathrm{~min})$; time difference $-5.1 \mathrm{~min}(95 \% \mathrm{CI}-5.9$ to -4.3$)$ and $-4.3 \min (95 \% \mathrm{CI}-5.1$ to -3.6$)$. Mean times for acquisition and interpretation and between-protocol time differences are shown in Appendix 8. We found no significant correlation between expert LUS congestion score evolution and temporal change in NT-proBNP $(\rho=0.16$, $P=0.37$ for 8 -point, $\rho=0.28, P=0.09$ for 28-point), body weight $(\rho=0.22, P=0.18$ for 8-point, $\rho=-0.21, P=0.20$ for 28-point) or EVEREST score ( $\rho=0.15, P=0.37$ for 8-point, $\rho=-0.11, P=0.53$ for 28-point). Results and dot plots are presented in Appendix 9. Modest albeit significant correlation was observed between LUS and NT-proBNP values, when analysed separately at admission and at follow-up (Appendix 10, 11).

Interestingly, the length of hospital stay seems to be lower in 6 patients with no detectable congestion on expert 8-point aLUS (i.e. $<2 / 8$ positive points: median 4.5 days, IQR 4 to 5) when compared to 37 patients with mild to severe congestion (i.e. $\geq 2 / 8$ positive points: median 13 days, IQR 7 to 19 , $P=0.015$ ). Additionally, a trend to lower rates of 30 - and 60 day readmission and mortality was observed in patients without congestion on expert 8-point fLUS as presented in Table 4. Results were similar when using expert 28-point LUS.

\section{Discussion}

In this prospective comparative study, pulmonary congestion was detected by LUS in the majority of patients at admission and decreased at follow-up. Whereas significant congestion was detected in a greater proportion of patients by both experts and novices when using 28-point protocol, the 8 -point protocol required significantly less time for imaging 
Table 2 Key clinical and biological features of patients at admission and follow-up

\begin{tabular}{|c|c|c|c|c|}
\hline & $N=43$ & Admission & $N=39$ & Follow-up \\
\hline EVEREST score (total), median (IQR) & 39 & $8(5-10)$ & 38 & $3(2-6)$ \\
\hline Dyspnea, $n(\%)$ & 41 & & 39 & \\
\hline None & & $5(12)$ & & $22(57)$ \\
\hline Seldom & & $12(29)$ & & $11(28)$ \\
\hline Frequent & & $17(42)$ & & $6(15)$ \\
\hline Continuous & & $7(17)$ & & $0(0)$ \\
\hline Orthopnea, $n(\%)$ & 41 & & 39 & \\
\hline None & & $17(42)$ & & $20(72)$ \\
\hline Seldom & & $11(27)$ & & $10(26)$ \\
\hline Frequent & & $7(17)$ & & $1(2)$ \\
\hline Continuous & & $6(14)$ & & $0(0)$ \\
\hline Fatigue, $n(\%)$ & 41 & & 39 & \\
\hline None & & $5(12)$ & & $9(23)$ \\
\hline Seldom & & $9(22)$ & & $23(59)$ \\
\hline Frequent & & $21(51)$ & & $5(13)$ \\
\hline Continuous & & $6(15)$ & & $2(5)$ \\
\hline $\mathrm{JVD}, n(\%)$ & 39 & & 38 & \\
\hline$\leq 6 \mathrm{~cm} \mathrm{H} 2 \mathrm{O}$ & & $20(51)$ & & $26(68)$ \\
\hline $6-9$ & & $11(28)$ & & $9(24)$ \\
\hline $10-15$ & & $7(18)$ & & $3(8)$ \\
\hline$\geq 15$ & & $1(3)$ & & $0(0)$ \\
\hline Rales, $n(\%)$ & 41 & & 39 & \\
\hline None & & $8(20)$ & & $19(49)$ \\
\hline Bases & & $25(61)$ & & $18(46)$ \\
\hline Up to $<50 \%$ & & $5(12)$ & & $2(5)$ \\
\hline$\geq 50 \%$ & & $3(7)$ & & $0(0)$ \\
\hline Oedema, $n(\%)$ & 41 & & 39 & \\
\hline Absent/trace & & $9(22)$ & & $22(56)$ \\
\hline Slight & & $11(27)$ & & $11(28)$ \\
\hline Moderate & & $9(22)$ & & $3(8)$ \\
\hline Marked & & $12(29)$ & & $3(8)$ \\
\hline Body weight, median (IQR), $\mathrm{kg}$ & 42 & $82.8(75.1-93.0)$ & 41 & $78.8(72.3-92.6)$ \\
\hline Serum NT-proBNP, median (IQR), ng/L & 43 & $4618(1775-8066)$ & 41 & $2587(901-4295)$ \\
\hline
\end{tabular}

$J V D$ means jugular vein distention
Table 3 Interobserver agreement at admission and follow-up in all patients and according to body mass index

\begin{tabular}{lll}
\hline & \multicolumn{2}{l}{ Weighted kappa (95\% CI) } \\
\cline { 2 - 3 } & Admission (aLUS) & Follow-up (fLUS) \\
\hline All patients $(n=43)$ & & \\
8-point protocol & $0.54(0.35-0.74)$ & $0.62(0.47-0.77)$ \\
28-point protocol & $0.51(0.31-0.71)$ & $0.41(0.25-0.57)$ \\
$P$ value for comparison & 0.74 & 0.13 \\
Non obese patients $(n=25)$ & & \\
8-point protocol & $0.61(0.37-0.86)$ & $0.62(0.43-0.81)$ \\
28-point protocol & $0.59(0.36-0.82)$ & $0.34(0.10-0.58)$ \\
Obese patients $(n=18)$ & & \\
8-point protocol & $0.43(0.11-0.75)$ & $0.57(0.36-0.77)$ \\
28-point protocol & $0.38(0.04-0.72)$ & $0.43(0.22-0.64)$ \\
\hline
\end{tabular}

and interpretation. A previous study of 20 ICU patients showed a reduction in examination time with no significant reduction in B-lines detection when decreasing the number of scanning points from 28 to 8 or 6 [18]. In another recent multicentric study, the diagnostic value of several LUS protocols were compared in dyspnoeic ED patients. Onehundred-seventeen subjects underwent the 28-point protocol at admission. Four, 6- and 8-point protocols were derived post hoc by selecting part of the 28 recorded video clips. The eight-point protocol was associated with a significant increase in diagnostic accuracy in a subset of patients with an uncertain diagnosis following clinical assessment [19]. In this trial, however, results are exposed to bias due to protocols not being performed independently. Moreover, derivation of 8 from 28-point protocol prevented sonographers 
Table 4 In-hospital length of stay and early clinical outcomes according to lung ultrasonography

\begin{tabular}{|c|c|c|c|c|c|c|c|}
\hline \multirow{2}{*}{$\frac{\text { Admission expert LUS }}{\text { Degree of congestion }}$} & \multirow{2}{*}{$\begin{array}{l}\text { Total } \\
n=43\end{array}$} & \multicolumn{2}{|l|}{ 8-point protocol } & \multirow[t]{2}{*}{$P$ value } & \multicolumn{2}{|l|}{ 28-point protocol } & \multirow[t]{2}{*}{$P$ value } \\
\hline & & Absent $(n=6)$ & Mild to Severe $(n=37)$ & & Absent $(n=4)$ & Mild to Severe $(n=39)$ & \\
\hline $\begin{array}{l}\text { Length of stay, days, } \\
\text { median (IQR) }\end{array}$ & $13.0(5.0-17.0)$ & $4.5(4.0-5.0)$ & $13.0(7.0-19.0)$ & 0.02 & $8.0(3.5-12.5)$ & $13.0(6.0-19.0)$ & 0.21 \\
\hline \multicolumn{8}{|l|}{ Follow-up expert LUS } \\
\hline Degree of congestion & $N=38^{\mathrm{a}}$ & Absent $(n=13)$ & Mild to severe $(n=25)$ & & Absent $(n=11)$ & Mild to severe $(n=27)$ & \\
\hline $\begin{array}{l}\text { 30-day readmission } \\
\text { and mortality, } n(\%)\end{array}$ & $6(16)$ & $0(0)$ & $6(24)$ & 0.08 & $1(9)$ & $5(18)$ & 0.65 \\
\hline $\begin{array}{l}\text { 60-day readmission } \\
\text { and mortality, } n(\%)\end{array}$ & $9(24)$ & $1(8)$ & $8(32)$ & 0.13 & $2(18)$ & $7(26)$ & 1.00 \\
\hline
\end{tabular}

${ }^{\mathrm{a}}$ one patient died during the index hospitalisation

from exploring posteriorly to the mid-axillary line, where EVLW tends to cumulate in a semi-recumbent patients as shown in our study (Appendix 5) and in previous reports [10]. These results may, therefore, not be applicable in less congested subjects as in hospitalized AHF patients.

In both cited studies, only trained sonographers performed LUS. It is worth noting that, if interobserver agreement is generally considered substantial for LUS, most studies are based on post-hoc off-line review of video loops acquired by a unique expert sonographer [26]. Image acquisition could, however, be an important source of variability, particularly for pairs of expert-novice sonographers. In our study LUS was performed and interpreted real-time independently by both experts and novices and we observed moderate to substantial agreement with no significant difference between protocols. Our findings are concordant with a prior report of 91 ED dyspneic patients undergoing a 10-zones LUS performed bedside by pairs of expert-novice sonographers, observing moderate agreement in counting B-lines (ICC 0.59) [27].

Early publications claimed that a 28-point protocol required $<3 \mathrm{~min}$ [28]; in contrast to subsequent reports suggesting 5 to 15 min was nearer the case thus rendering it impractical for daily clinical practice, especially in emergency settings [16]. In the present study the 28-point protocol took an average of 6 and 9 min for experts and novices, respectively; scanning time was reduced by more than $50 \%$ with 8-point protocol. In clinical practice LUS is interpreted during acquisition. The separating of image acquisition and interpretation, due to the study design, may have artificially overestimated total time.

Despite 28-point LUS being feasible in all patients, onefifth of scanned points was invalid due to visualization of extra-pulmonary structures. With the 8-point protocol, imaging was possible in $100 \%$ of scanning points. This and the fact that the same regions of thorax are explored may explain the limited loss of information when using reduced scanning point protocols.
This study showed modest albeit significant correlation between LUS and NT-proBNP values at admission and follow-up. No significant correlation, however, was highlighted between the decrease of LUS congestion and clinical evolution, weight loss and NT-proBNP decline, irrespective of the protocol used. Similarly, a previous study did not find significant correlation between admission-discharge delta BNP and delta LUS [10]. In contrast, in this study, delta LUS correlates significantly with delta clinical congestion score $(r=0.49, P<0.05)$. When compared to this study, our patients had lower clinical congestion at admission (median value of $8 / 10$ versus $8 / 18$, respectively), and lower decrease at follow-up (-89\% versus - 63\%), explaining differences in results.

Clinical appreciation of volemia is difficult, residual congestion at discharge is frequent and seems to be a key factor in hospital readmissions, even at subclinical stage [8]. In our study, rales were judged absent in one quarter of patients who still had significant LUS congestion at follow-up. Interestingly, patients with persistent congestion on expert 8 -point fLUS (i.e. $\geq 2 / 8$ positive points) had higher rate of post-discharge mortality and readmission at 30 days $(24 \%$ versus $0 \%$ ) and 60 days (32\% versus $8 \%$, Table 4 ) indicating the prognostic value of LUS congestion on early clinical outcomes, as previously shown in hospitalized and ambulatory heart failure patients [13, 29]. Interestingly, in a previous study 8 - and 28-point LUS similarly predict clinical outcomes [30]. Complete LUS decongestion before discharge may, therefore, be a valuable target to improve early clinical outcomes. If recent studies suggest that an ambulatory LUS-driven decongestion strategy may reduce unplanned urgent visits or hospital admissions in chronic heart failure patients [11, 12, 31], no data are currently available for AHF inpatients.

This study has certain limitations. First, the collected sample for this exploratory study was modest, due to recruitment interruption during the COVD-19 pandemic, and the precision of kappa statistics was lower than planned, ranging from \pm 0.2 at admission to \pm 0.15 at follow-up, instead 
of the planned \pm 0.12 . Second, the 8-point protocol used in this study was not mentioned in the international guidelines on lung ultrasonography [17]. These guidelines have not been updated since 2012, whilst the 8-point protocol was introduced in the past decade $[23,24]$. Third, the exclusion of severely congested AHF patients (i.e. requiring ICU admission) may affect generalizability in that population. However, benefits of LUS in AHF are more marked when pulmonary congestion is moderate, and its clinical detection becomes challenging. Additionally, interobserver concordance is more easily achieved for extremes (i.e. high and low number of B-lines) than for intermediate levels of congestion [27]. Finally, sonographers could not be blinded to patients and this may have influenced LUS interpretation.

\section{Conclusions}

In spite of its limitations, the present study has succeeded in bringing two essential answers to the ongoing LUS protocol debate. There is moderate to substantial agreement between experts and novices after a short, structured training period, when LUS is executed and interpreted independently at the bedside. Further trials should, in our opinion, include novices amongst study sonographers. Moreover, in AHF inpatients we found no benefit in terms of reproducibility in using an exhaustive 28-point protocol which required more than double the time in image acquisition and interpretation. Future research and clinical efforts could be concentrated in LUS protocols with limited scanning points.

Supplementary Information The online version contains supplementary material available at https://doi.org/10.1007/s11739-022-02943-9.

Acknowledgements This study would not have been possible without the passionate contribution of all the members of the IcarUS study group, from the General Internal Medicine of Geneva University Hospitals, Geneva, Switzerland (Arnaud Dominati, Laetitia Dubouchet, Sebastian Carballo, Matteo Coen, Florence Coste, Amelia Goncalves, Alexandre Guillermin, Olivier Grosgurin, Myriam Kaddour, Flora Koegler, Antonio Leidi, Alexandre Leszek, Tamara Mann, Christophe Marti, Sofia Merlo, Marine Monney, Guillaume Soret, Jean-Luc Reny, and Jérôme Stirnemann) and of all patients included.

Funding Open access funding provided by University of Geneva. This work was supported by the Edmond J SAFRA Foundation for Clinical Research. The funders had no role in the study.

\section{Declarations}

Conflict of interest The authors declare that they have no conflict of interest.

Human and animal rights statement and Informed consent The investigation conforms with the principles outlined in the Declaration of Helsinki and was approved by the local ethics committee (CCER
2019-01,596). Informed consent was obtained from all patients prior to inclusion.

Open Access This article is licensed under a Creative Commons Attribution 4.0 International License, which permits use, sharing, adaptation, distribution and reproduction in any medium or format, as long as you give appropriate credit to the original author(s) and the source, provide a link to the Creative Commons licence, and indicate if changes were made. The images or other third party material in this article are included in the article's Creative Commons licence, unless indicated otherwise in a credit line to the material. If material is not included in the article's Creative Commons licence and your intended use is not permitted by statutory regulation or exceeds the permitted use, you will need to obtain permission directly from the copyright holder. To view a copy of this licence, visit http://creativecommons.org/licenses/by/4.0/.

\section{References}

1. Maggioni AP, Dahlstrom U, Filippatos G, Chioncel O, Crespo Leiro M, Drozdz J et al (2013) EUR observational research programme: regional differences and 1-year follow-up results of the heart failure pilot survey (ESC-HF Pilot). Eur J Heart Fail 15(7):808-817

2. Donze J, Lipsitz S, Bates DW, Schnipper JL (2013) Causes and patterns of readmissions in patients with common comorbidities: retrospective cohort study. BMJ 347:f7171

3. Dharmarajan K, Hsieh AF, Kulkarni VT, Lin Z, Ross JS, Horwitz LI et al (2015) Trajectories of risk after hospitalization for heart failure, acute myocardial infarction, or pneumonia: retrospective cohort study. BMJ 350:h411

4. Ponikowski P, Voors AA, Anker SD, Bueno H, Cleland JG, Coats AJ et al (2016) ESC Guidelines for the diagnosis and treatment of acute and chronic heart failure: the task force for the diagnosis and treatment of acute and chronic heart failure of the european society of cardiology (ESC). developed with the special contribution of the heart failure association (HFA) of the ESC. Eur J Heart Fail 18(8):891-975

5. Ambrosy AP, Pang PS, Khan S, Konstam MA, Fonarow GC, Traver B et al (2013) Clinical course and predictive value of congestion during hospitalization in patients admitted for worsening signs and symptoms of heart failure with reduced ejection fraction: findings from the EVEREST trial. Eur Heart $\mathbf{J}$ 34(11):835-843

6. Leidi A, Rouyer F, Marti C, Reny JL, Grosgurin O (2020) Point of care ultrasonography from the emergency department to the internal medicine ward: current trends and perspectives. Intern Emerg Med 15(3):395-408

7. Maw AM, Hassanin A, Ho PM, McInnes MDF, Moss A, JuarezColunga E et al (2019) Diagnostic accuracy of point-of-care lung ultrasonography and chest radiography in adults with symptoms suggestive of acute decompensated heart failure: a systematic review and meta-analysis. JAMA Netw Open 2(3):e190703

8. Rivas-Lasarte M, Maestro A, Fernandez-Martinez J, LopezLopez L, Sole-Gonzalez E, Vives-Borras M et al (2020) Prevalence and prognostic impact of subclinical pulmonary congestion at discharge in patients with acute heart failure. Esc Heart Fail 7(5):2621-2628

9. Miglioranza MH, Gargani L, Sant'Anna RT, Rover MM, Martins VM, Mantovani A et al (2013) Lung ultrasound for the evaluation of pulmonary congestion in outpatients: a comparison with clinical assessment, natriuretic peptides, and echocardiography. JACC Cardiovasc Imaging 6(11):1141-1151 
10. Volpicelli G, Caramello V, Cardinale L, Mussa A, Bar F, Frascisco MF (2008) Bedside ultrasound of the lung for the monitoring of acute decompensated heart failure. Am J Emerg Med 26(5):585-591

11. Marini C, Fragasso G, Italia L, Sisakian H, Tufaro V, Ingallina $G$ et al (2020) Lung ultrasound-guided therapy reduces acute decompensation events in chronic heart failure. Heart 106(24):1934-1939

12. Rivas-Lasarte M, Alvarez-Garcia J, Fernandez-Martinez J, Maestro A, Lopez-Lopez L, Sole-Gonzalez E et al (2019) Lung ultrasound-guided treatment in ambulatory patients with heart failure: a randomized controlled clinical trial (LUS-HF study). Eur J Heart Fail 21(12):1605-1613

13. Platz E, Campbell RT, Claggett B, Lewis EF, Groarke JD, Docherty KF et al (2019) Lung ultrasound in acute heart failure: prevalence of pulmonary congestion and short- and long-term outcomes. JACC Heart Fail 7(10):849-858

14. Coiro S, Porot G, Rossignol P, Ambrosio G, Carluccio E, Tritto I et al (2016) Prognostic value of pulmonary congestion assessed by lung ultrasound imaging during heart failure hospitalisation: a two-centre cohort study. Sci Rep 6:39426

15. Palazzuoli A, Ruocco G, Beltrami M, Nuti R, Cleland JG (2018) Combined use of lung ultrasound, B-type natriuretic peptide, and echocardiography for outcome prediction in patients with acute HFrEF and HFpEF. Clin Res Cardiol 107(7):586-596

16. Martindale JL (2016) Resolution of sonographic B-lines as a measure of pulmonary decongestion in acute heart failure. Am J Emerg Med 34(6):1129-1132

17. Volpicelli G, Elbarbary M, Blaivas M, Lichtenstein DA, Mathis G, Kirkpatrick AW et al (2012) International evidence-based recommendations for point-of-care lung ultrasound. Intensive Care Med 38(4):577-591

18. Cox EGM, Wiersema R, Wong A, van der Horst ICC, Group SS (2020) Six versus eight and twenty-eight scan sites for B-line assessment: differences in examination time and findings. Intensive Care Med 46(5):1063-1064

19. Buessler A, Chouihed T, Duarte K, Bassand A, Huot-Marchand M, Gottwalles Y et al (2020) Accuracy of several lung ultrasound methods for the diagnosis of acute heart failure in the ed a multicenter prospective study. Chest 157(1):99-110

20. Russell FM, Ferre R, Ehrman RR, Noble V, Gargani L, Collins SP et al (2020) What are the minimum requirements to establish proficiency in lung ultrasound training for quantifying B-lines? Esc Heart Fail 7(5):2941-2947

21. Mozzini C, Fratta Pasini AM, Garbin U, Cominacini L (2016) Lung ultrasound in internal medicine: training and clinical practice. Crit Ultrasound J 8(1): 10
22. Platz E, Jhund PS, Girerd N, Pivetta E, McMurray JJV, Peacock WF et al (2019) Expert consensus document: reporting checklist for quantification of pulmonary congestion by lung ultrasound in heart failure. Eur J Heart Fail 21(7):844-851

23. Pivetta E, Goffi A, Lupia E, Tizzani M, Porrino G, Ferreri E et al (2015) Lung ultrasound-implemented diagnosis of acute decompensated heart failure in the ed a simeu multicenter study. Chest 148(1):202-210

24. Cibinel GA, Casoli G, Elia F, Padoan M, Pivetta E, Lupia E et al (2012) Diagnostic accuracy and reproducibility of pleural and lung ultrasound in discriminating cardiogenic causes of acute dyspnea in the emergency department. Intern Emerg Med 7(1):65-70

25. Gargani L, Pang PS, Frassi F, Miglioranza MH, Dini FL, Landi $P$ et al (2015) Persistent pulmonary congestion before discharge predicts rehospitalization in heart failure: a lung ultrasound study. Cardiovasc Ultrasound 13:40

26. Anderson KL, Fields JM, Panebianco NL, Jenq KY, Marin J, Dean AJ (2013) Inter-rater reliability of quantifying pleural b-lines using multiple counting methods. J Ultras Med 32(1):115-120

27. Gullett J, Donnelly JP, Sinert R, Hosek B, Fuller D, Hill H et al (2015) Interobserver agreement in the evaluation of B-lines using bedside ultrasound. J Crit Care 30(6):1395-1399

28. Frassi F, Gargani L, Gligorova S, Ciampi Q, Mottola G, Picano E (2007) Clinical and echocardiographic determinants of ultrasound lung comets. Eur J Echocardiogr 8(6):474-479

29. Platz E, Merz AA, Jhund PS, Vazir A, Campbell R, McMurray JJ (2017) Dynamic changes and prognostic value of pulmonary congestion by lung ultrasound in acute and chronic heart failure: a systematic review. Eur J Heart Fail 19(9):1154-1163

30. Cogliati C, Casazza G, Ceriani E, Torzillo D, Furlotti S, Bossi I et al (2016) Lung ultrasound and short-term prognosis in heart failure patients. Int J Cardiol 218:104-108

31. Araiza-Garaygordobil D, Gopar-Nieto R, Martinez-Amezcua P, Cabello-Lopez A, Alanis-Estrada G, Luna-Herbert A et al (2020) A randomized controlled trial of lung ultrasound-guided therapy in heart failure (CLUSTER-HF study). Am Heart J 227:31-39

Publisher's Note Springer Nature remains neutral with regard to jurisdictional claims in published maps and institutional affiliations. 